\title{
The Effect of Budesonide/Formoterol Pressurized Metered-Dose Inhaler on Predefined Criteria for Worsening Asthma in Four Different Patient Populations with Asthma
}

\author{
Kevin R. Murphy, ${ }^{1}$ Tom Uryniak, ${ }^{2}$ Ubaldo J. Martin ${ }^{2}$ and James Zangrilli ${ }^{2}$ \\ 1 Boys Town National Research Hospital, Omaha, NE, USA \\ 2 AstraZeneca LP, Wilmington, DE, USA
}

\section{Abstract}

Background: Previous studies have shown disparities between Black and Hispanic patients compared with other populations in response to asthma medications.

Objective: The aim of this analysis was to assess the effect of budesonide/ formoterol pressurized metered-dose inhaler (BUD/FM pMDI) and BUD on predefined criteria for asthma worsening, an asthma control metric generally aligned with definitions of moderate asthma exacerbations, across four different populations.

Methods: Data were from four 12-week, randomized, double-blind, US studies of BUD/FM pMDI treatment in patients aged 12 years or older with varying asthma severities and of varying races. Predefined asthma events and withdrawals due to predefined events were assessed as secondary study endpoints. Study I (NCT00651651) includes data from predominantly White patients with mild to moderate asthma who were randomized to BUD/FM pMDI $160 / 9 \mu \mathrm{g}$ twice daily (bid; $\mathrm{n}=123)$ or BUD pMDI $160 \mu \mathrm{g}$ bid $(\mathrm{n}=121)$. Study II (NCT00652002) includes data from predominantly White patients with moderate to severe asthma who were randomized to BUD/FM pMDI $320 / 9 \mu \mathrm{g}$ bid $(\mathrm{n}=124)$ or BUD pMDI $320 \mu \mathrm{g}$ bid $(\mathrm{n}=109)$. Study III (NCT00702325) included self-reported Black patients with moderate to severe asthma who were randomized to BUD/FM pMDI 320/9 $\mu$ g bid $(n=153)$ or BUD dry powder inhaler $360 \mu \mathrm{g}$ bid $(\mathrm{n}=148)$. Study IV (NCT00419757) included self-reported Hispanic patients with moderate to severe asthma who were randomized to BUD/FM pMDI 320/9 $\mu \mathrm{g}$ bid $(\mathrm{n}=127)$ or BUD pMDI $320 \mu \mathrm{g}$ bid $(\mathrm{n}=123)$. Patients were to be withdrawn from the studies if they developed an asthma event, as determined by predefined criteria, except for night-time awakenings, where withdrawal was left to the study physician's judgment. 
Results: Overall, fewer patients in the studies (study I, II, III, and IV, respectively) experienced $\geq 1$ asthma event in the BUD/FM group (18.7\%, $29.8 \%, 37.3 \%, 25.2 \%)$ versus the BUD group $(21.5 \%, 44.0 \%, 45.3 \%, 31.7 \%)$; only study II results showed a statistically significant difference between treatments. Fewer patients with moderate to severe asthma (studies II, III, and IV) were withdrawn due to $\geq 1$ asthma event in the BUD/FM group $(10.5 \%, 11.8 \%, 3.1 \%$, respectively) than in the BUD group $(20.2 \%, 18.9 \%$, $6.5 \%$, respectively); however, percentages were similar in the BUD/FM (7.3\%) and BUD (6.6\%) groups in patients with mild to moderate asthma (study I).

Conclusions: Predefined asthma event rates were numerically or significantly lower for patients with asthma receiving BUD/FM pMDI versus BUD, regardless of race or disease severity. Differences between the BUD/FM pMDI and BUD groups were smaller in patients with mild to moderate asthma than in those with moderate to severe asthma, most likely because patients with milder disease had lower asthma event rates. Overall, these findings support the efficacy of BUD/FM pMDI in achieving asthma control in patients with moderate to severe asthma.

\section{Introduction}

Asthma disproportionately affects racial and ethnic populations. In the US in 2006, the ageadjusted, asthma-related mortality rates were approximately 3 times higher in non-Hispanic Blacks than in non-Hispanic Whites and Hispanics. ${ }^{[1]} \mathrm{Al}-$ though typical safety and efficacy studies are underpowered or too short in duration to make definitive conclusions regarding severe asthma exacerbations (i.e. those requiring systemic corticosteroids), important insight into the efficacy of medications can be gained from analyzing related moderate exacerbation events characterized by a sustained loss of asthma control (beyond normal day-today variation) that does not meet the definition of a severe exacerbation. ${ }^{[2]}$ For the purpose of asthma research protocol development, moderate exacerbation events have been captured using various terminology, such as asthma deterioration, ${ }^{[3]}$ asthma worsenings, ${ }^{[4]}$ and asthma events. ${ }^{[5]}$ Few US studies have evaluated the safety and efficacy of an inhaled corticosteroid (ICS)/long-acting $\beta_{2^{-}}$ adrenergic agonist (LABA) combination therapy in Black or Hispanic patients with asthma. The efficacy of budesonide/formoterol (BUD/FM) pressurized metered-dose inhaler (pMDI) has been evaluated in randomized, double-blind studies in predominantly White patients with mild to moderate asthma ${ }^{[6]}$ and predominantly White, ${ }^{[5]}$ Black, ${ }^{[7]}$ and Hispanic ${ }^{[8]}$ patients with moderate to severe asthma. Results for a predefined asthma event definition, which encompass moderate to severe asthma deteriorations, are presented as these findings have not been presented previously in detail or compared across patient populations.

\section{Methods}

Table I includes a brief summary of the studies that were included in this exploratory analysis. Additional details of the individual studies, including study design and methods, have been previously described. ${ }^{[5-8]}$ This analysis of predefined asthma events (table II) includes data from patients aged $\geq 12$ years with asthma who were enrolled in randomized, double-blind, 12-week US studies that differed according to baseline asthma severity and/or race or ethnicity (table III). Only the BUD/FM and BUD treatment arms, which were common to all four studies, are presented; these studies were not originally powered for comparison of asthma events.

Statistical methods for this analysis are similar to those described previously. ${ }^{[5-8]}$ Comparisons among treatment groups of percentages of patients 
Table I. Study treatments and entry criteria ${ }^{[5-8]}$

\begin{tabular}{|c|c|c|c|c|}
\hline Study & Run-in & Interventions & Race & Asthma severity \\
\hline$\overline{l(N C T 00651651)^{[6]}}$ & Placebo pMDI bid & $\begin{array}{l}\text { BUD/FM pMDI } 160 / 9 \mu \mathrm{g}(\mathrm{n}=123) \\
\text { BUD pMDI } 160 \mu \mathrm{g}(\mathrm{n}=121) \\
\text { FM DPI } 9 \mu \mathrm{g}(\mathrm{n}=114) \\
\text { Placebo }(\mathrm{n}=122)\end{array}$ & Not specified & Mild to moderate \\
\hline II (NCT00652002) ${ }^{[5]}$ & BUD pMDI $160 \mu \mathrm{g}$ bid & $\begin{array}{l}\text { BUD/FM pMDI } 320 / 9 \mu \mathrm{g}(\mathrm{n}=124) \\
\text { BUD pMDI } 320 \mu \mathrm{g}(\mathrm{n}=109) \\
\text { BUD pMDI } 320 \mu \mathrm{g}+\text { FM DPI } 9 \mu \mathrm{g}(\mathrm{n}=115) \\
\text { FM DPI } 9 \mu \mathrm{g}(\mathrm{n}=123) \\
\text { Placebo }(\mathrm{n}=125)\end{array}$ & Not specified & Moderate to severe \\
\hline III (NCT00702325) ${ }^{[7]}$ & BUD DPI $180 \mu \mathrm{g}$ bid & $\begin{array}{l}\text { BUD/FM pMDI 320/9 } \mu \mathrm{g}(\mathrm{n}=156) \\
\text { BUD DPI } 360 \mu \mathrm{g}(\mathrm{n}=155)\end{array}$ & Self-reported Black ${ }^{a}$ & Moderate to severe \\
\hline IV (NCT00419757) ${ }^{[8]}$ & BUD pMDI $160 \mu \mathrm{g}$ bid & $\begin{array}{l}\text { BUD/FM pMDI 320/9 } \mu \mathrm{g}(\mathrm{n}=127) \\
\text { BUD pMDI } 320 \mu \mathrm{g}(\mathrm{n}=123)\end{array}$ & Self-reported Hispanic ${ }^{b}$ & Moderate to severe \\
\hline
\end{tabular}

who experienced $\geq 1$ predefined asthma event and of percentages of patients who withdrew because of such an event were performed by $\chi^{2}$ test (study I) or Cochran-Mantel-Haenszel test with adjustment for treatment (studies III and IV) and ICS dose (medium or high; studies II, III, and IV) at study entry.

\section{Results}

Baseline demographics were similar across studies (table II). As expected, patients with mild to moderate asthma had better pulmonary function than those with moderate to severe asthma.

The percentage of patients with moderate to severe asthma who experienced $\geq 1$ asthma event was lower in the BUD/FM groups versus the BUD group, with statistically significant differences observed in study II $(p<0.05)$ [figure 1]. In all studies, the most commonly met predefined criterion was night-time awakening. The predefined criterion of clinical exacerbation included the following subcategories that were not mutually exclusive:

- study I (BUD/FM: one patient [one emergency department (ED) visit, one event of disallowed asthma medication use], BUD: three patients [one ED visit, three events of disallowed asthma medication use]);

- study II (BUD/FM: seven patients [three ED visits, seven events of disallowed asthma medication use], BUD: five patients [one ED visit, four events of disallowed asthma medication use]);

- study III (BUD/FM: three patients [two events of disallowed asthma medication use, one event of nebulized bronchodilator use, three events of oral corticosteroid use], BUD: three patients [one ED visit, three events of disallowed asthma medication use, one event of nebulized bronchodilator use, and three events of oral corticosteroid use]);

- study IV (BUD/FM: seven patients [two ED visits, two hospitalizations - one due to multiple

Table II. Predefined criteria for asthma events ${ }^{[5-8]}$

1. Decrease in morning predose $\mathrm{FEV}_{1} \geq 20 \%$ from randomization or a decrease to $<40 \%$ a of predicted normal

2. $\geq 12$ Actuations of albuterol/day on $\geq 3$ days within a 7-day period

3. Decrease in morning PEF $\geq 20 \%$ from baseline on $\geq 3$ days within a 7-day period

4. $\geq 2$ Nights with an awakening due to asthma requiring rescue medication within any 7-day period ${ }^{\mathrm{b}}$

5. Clinical exacerbation requiring emergency treatment, hospitalization, or use of an asthma medication not allowed by the protocol

a For study I, $<45 \%$.

b Patients experiencing only the night-time awakening criterion could remain in the study if the investigator determined that they were clinically well and stable.

$\mathbf{F E V}_{\mathbf{1}}=$ forced expiratory volume in 1 second; $\mathbf{P E F}=$ peak expiratory flow. 




significant/active comorbidities and one due to viral infection, seven events of disallowed asthma medication use], BUD: two patients [two events of disallowed medication use]).

Overall, withdrawal rates were lower in studies I and IV than in studies II and III (figure 1). The percentage of patients with mild to moderate asthma (study I) who withdrew due to $\geq 1$ predefined asthma event was similar in the BUD/FM and BUD groups. Percentages of patients with moderate to severe asthma (studies II, III, and IV) who withdrew due to $\geq 1$ asthma event were numerically lower in the BUD/FM versus BUD groups, regardless of race. Additional results from the individual studies have been previously described. ${ }^{[5-8]}$

\section{Conclusions}

Predefined asthma events are increasingly being utilized in clinical research studies as a sensitive composite control metric. An asthma event metric encompassing measures of pulmonary function, symptoms, rescue medication use, and the need for additional medications was investigated in the present analysis. While individual studies were not powered for statistical analyses, predefined asthma event rates in four 12-week, randomized studies consistently showed numerical or significant differences favoring BUD/FM pMDI over BUD across White, Black, and Hispanic patients, regardless of disease severity. Notably, the results of this analysis showing similar predefined asthma event rates among patients of differing racial backgrounds is consistent with the primary analyses showing the efficacy of BUD/ FM pMDI in Blacks ${ }^{[7]}$ and Hispanics, ${ }^{[8]}$ as well as a study demonstrating the efficacy of ICS/LABA in Blacks. ${ }^{[9]}$ Additional discussion of findings and limitations of the individual studies have been previously discussed. ${ }^{[5-8]}$ Differences between the BUD/FM pMDI and BUD groups were smaller in patients with mild to moderate asthma than in patients with moderate to severe asthma, most likely because patients with milder disease had overall lower asthma event rates. These data further support the efficacy of BUD/FM pMDI in achieving asthma control in patients with moderate to severe asthma, regardless of race. 


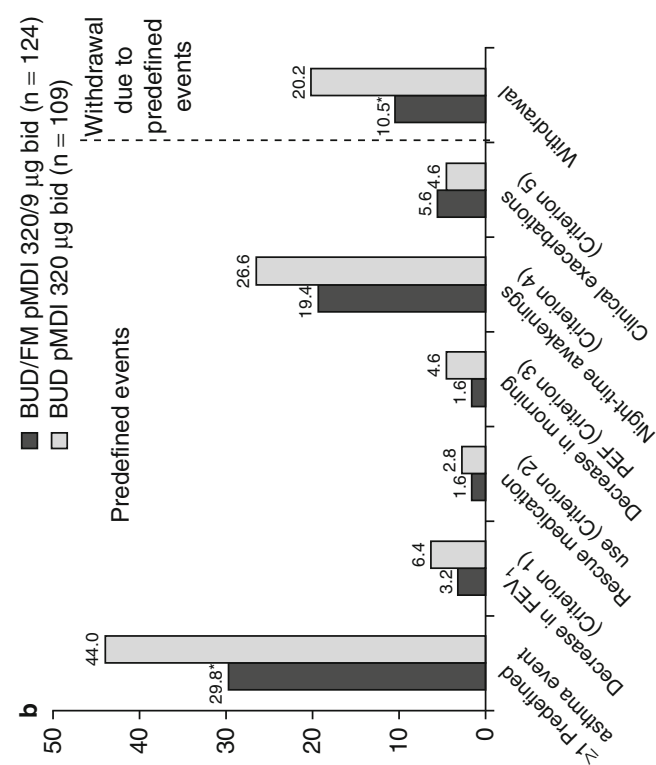

(\%) słue!led

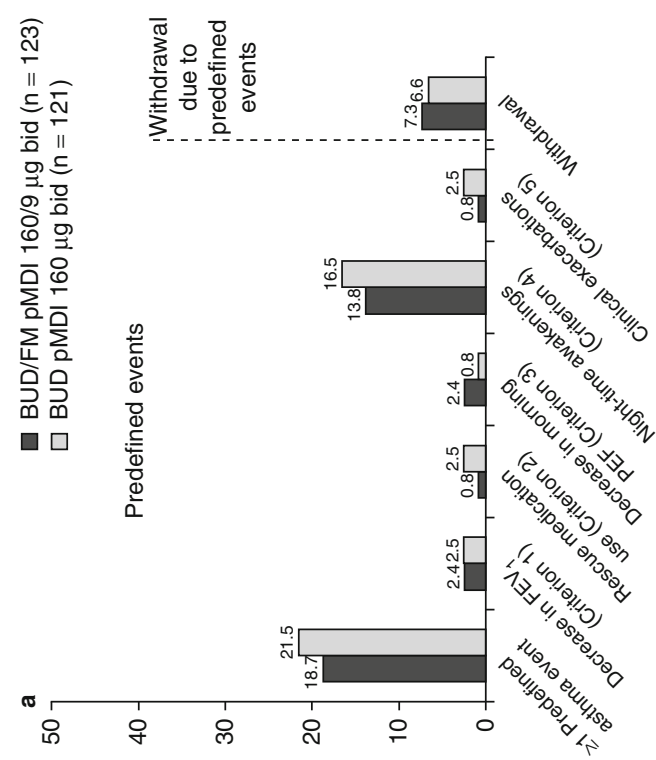

(\%) słuə!̣ed

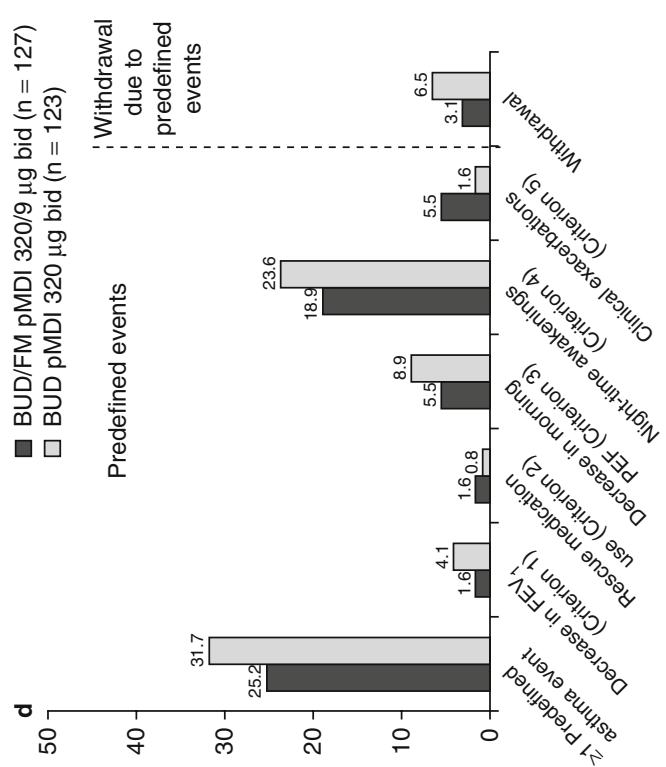

(\%) słue!̣ed

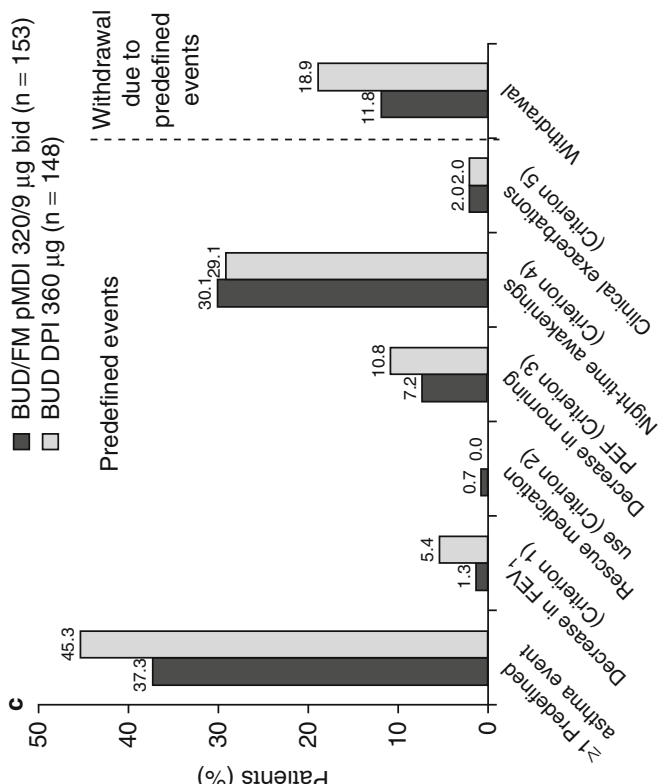

(\%) słuə!̣ed

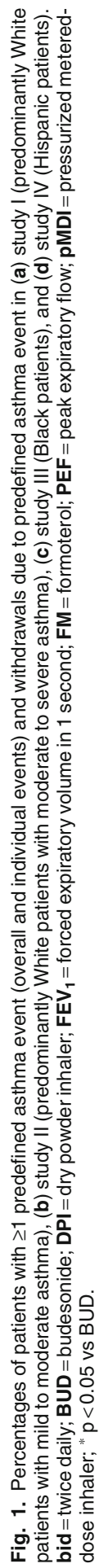




\section{Acknowledgments}

This study was supported by AstraZeneca LP, Wilmington, DE, USA. Medical writing services, provided by Lisa Feder, PhD (Scientific Connexions, Newtown, PA, USA), were funded by AstraZeneca LP.

K.R. Murphy, T. Uryniak, U.J. Martin, and J. Zangrilli made substantial contributions to the analysis and interpretation of data, drafted and revised the manuscript critically for important intellectual content, and provided final approval of the version to be published.

K.R. Murphy is a(n) consultant and advisor to and has received lecture fees and grants from AstraZeneca LP. T. Uryniak, U.J. Martin, and J. Zangrilli are shareholders and employees of AstraZeneca LP.

\section{References}

1. American Lung Association. Trends in asthma morbidity and mortality. July 2011 [online]. Available from URL: http:// www.lungusa.org/finding-cures/our-research/trend-reports/ asthma-trend-report.pdf [Accessed 2011 Oct 21]

2. Reddel HK, Taylor DR, Bateman ED, et al. An official American Thoracic Society/European Respiratory Society statement: asthma control and exacerbations. Am J Respir Crit Care Med 2009; 180 (1): 59-99

3. Nathan RA, Nolte H, Pearlman DS, P04334 Study Investigators. Twenty-six-week efficacy and safety study of mometasone furoate/formoterol $200 / 10 \mu \mathrm{g}$ combination treatment in patients with persistent asthma previously receiving medium-dose inhaled corticosteroids. Allergy Asthma Proc 2010; 31 (4): 269-79

4. Kavuru M, Melamed J, Gross G, et al. Salmeterol and fluticasone propionate combined in a new powder inhalation device for the treatment of asthma: a randomized, double- blind, placebo-controlled trial. J Allergy Clin Immunol 2000; 105 (6 Pt 1): 1108-16

5. Noonan M, Rosenwasser LJ, Martin P, et al. Efficacy and safety of budesonide and formoterol in one pressurised metered-dose inhaler in adults and adolescents with moderate to severe asthma: a randomised clinical trial. Drugs 2006; 66 (5): 2235-54

6. Corren J, Korenblat PE, Miller CJ, et al. Twelve-week, randomized, placebo-controlled, multicenter study of the efficacy and tolerability of budesonide and formoterol in one metered-dose inhaler compared with budesonide alone and formoterol alone in adolescents and adults with asthma. Clin Ther 2007 May; 29 (5): 823-43

7. Spector SL, Martin UJ, Uryniak T, et al. Budesonide/ formoterol pressurized metered dose inhaler versus budesonide: a randomized controlled trial in Black patients with asthma. J Asthma 2012; 49 (1): 70-7

8. Zangrilli J, Mansfield LE, Uryniak T, et al. Efficacy of budesonide/formoterol pressurized metered-dose inhaler versus budesonide pressurized metered-dose inhaler alone in Hispanic adults and adolescents with asthma: a randomized, controlled trial. Ann Allergy Asthma Immunol 2011; 107 (3): $258-65$

9. Bailey W, Castro M, Matz J, et al. Asthma exacerbations in African Americans treated for 1 year with combination fluticasone propionate and salmeterol or fluticasone propionate alone. Curr Med Res Opin 2008; 24 (6): 1669-82

Correspondence: Kevin R. Murphy, MD, Boys Town National Research Hospital, Allergy, Asthma \& Pediatric Pulmonology, 14080 Hospital Road, Boys Town, NE 68010, USA.

E-mail: kevin.murphy@boystown.org 